Revue

Revue de l'histoire des religions

de Ihistoire

des religions

3 | 2016

Varia

Christophe GRELLARD, De la certitude volontaire. Débats nominalistes sur la foi à la fin du Moyen Âge

Paris, Publications de la Sorbonne (« La Philosophie à l'œuvre »), 2014

Bénédicte Sère

\title{
CpenEdition
}

Journals

Édition électronique

URL : http://journals.openedition.org/rhr/8605

DOI : 10.4000/rhr.8605

ISSN : 2105-2573

Éditeur

Armand Colin

Édition imprimée

Date de publication : 1 septembre 2016

Pagination : 440-443

ISBN : $978-2-200-93061-5$

ISSN : 0035-1423

Référence électronique

Bénédicte Sère, "Christophe grelLARD, De la certitude volontaire. Débats nominalistes sur la foi à la fin du Moyen Âge », Revue de l'histoire des religions [En ligne], 3 | 2016, mis en ligne le 06 octobre 2016,

consulté le 22 septembre 2020. URL : http://journals.openedition.org/rhr/8605 ; DOI : https://doi.org/ $10.4000 /$ rhr.8605

Ce document a été généré automatiquement le 22 septembre 2020

Tous droits réservés 


\section{Christophe GRELLARD, De la certitude volontaire. Débats nominalistes sur la foi à la fin du Moyen Âge}

Paris, Publications de la Sorbonne (« La Philosophie à l'œuvre »), 2014

Bénédicte Sère

\section{RÉFÉRENCE}

Christophe GRELLARD, De la certitude volontaire. Débats nominalistes sur la foi à la fin du Moyen Âge, Paris, Publications de la Sorbonne (« La Philosophie à l'œuvre »), 2014, $24 \mathrm{~cm}, 160$ p., $19 €$, ISBN 978-2-85944-777-9.

1 Qu'est-ce que croire? Peut-on décider de croire ? Comment décider de croire ? Doit-on croire avec la raison ou avec la volonté ? Lorsque Christophe Grellard écrit une histoire des débats nominalistes sur la foi ou "certitude volontaire ", il s'inscrit dans le décor historiographique de ces dernières années, le sien et celui d'autres jeunes chercheurs. En 2005, en effet, il réfléchissait déjà sur Nicolas d'Autrécourt, nominaliste du XIV siècle, et sur son articulation entre croire et savoir. Récemment, il publie une étude sur le scepticisme, notamment chez Jean de Salisbury. Pascale Bermon avait, quant à elle, en 2006, centré sa réflexion sur les mêmes questions chez Grégoire de Rimini. L'année dernière, en 2013, c'est Monica Brînzei qui, en éditant les Principia du commentaire des Sentences de Pierre d'Ailly, donne à méditer sur la doctrine de l'assentiment et de la certitude de la foi. En cette même année 2013, paraît chez Vrin le volume collectif dirigé par Laurent Jaffro et intitulé Croit-on comme on veut? Histoire d'une controverse. C'est dire l'actualité du thème dont les pionniers en leur temps - Lucien Febvre sur Le problème de l'incroyance et Michel de Certeau sur La faiblesse de croire - avaient déjà brossé les enjeux.

2 L'objet des investigations de Christophe Grellard reste circonscrit: il s'agit pour lui d'étudier la perception doctrinale de l'acte de foi chez les penseurs nominalistes, 
quatre précisément : Guillaume d'Ockham, Robert Holcot, Pierre d'Ailly et Jean Mair. En amont (chapitre premier), l'auteur esquisse une sorte de généalogie des doctrines autour de l'acte de foi, d'Augustin à saint Thomas et à Buridan. L'idée est de rappeler la place centrale accordée à la volonté dans le système augustinien et sa relecture par Thomas d'Aquin au XIII ${ }^{\mathrm{e}}$ siècle, lequel, fort d'une théorie aristotélicienne des vertus, insiste sur l'acte de foi comme résultat d'une coopération entre l'intellect et la volonté. À l'occasion de ces débats, la foi est distinguée du savoir, de l'opinion et de la crédulité, mettant en jeu les notions de vérité, fermeté, certitude et évidence. De même, la place des autorités et des témoignages qui fondent l'assentiment de foi est centrale.

Suit le cœur de la démonstration en trois temps et en trois chapitres. Au premier temps (chapitre 2), Guillaume d'Ockham se pose en faveur d'un fondement volontaire de la foi. Plus précisément, il travaille, en amont de la décision de foi, sur l'autorité épistémique qui garantit le témoignage dont dépend l'acte de foi et induit une première adhésion décisive pour la suite : c'est le primum creditum, cette décision initiale de la volonté libre qui donne un assentiment sans évidence et sur témoignage. Pour le dire autrement, le premier acte de foi est un acte de la pure volonté libre qui se décide sans motif face à une proposition indécidable. Cet acte de volonté libre se prononce, non pas sur le contenu - ni évident ni décidable - mais sur la confiance accordée à une autorité ou à un témoignage : «C'est au magistère de l'Église ou au témoignage des apôtres que je décide de croire plus qu'au dogme de l'Incarnation ou à la Trinité, la croyance en ces dogmes étant, en quelque sorte, seconde » (p.73). Et l'auteur de parler ainsi d'un « volontarisme doxastique » (p. 74), limité certes mais intransigeant, surtout exclusif de tout motif rationnel de crédibilité, enfin atypique voire exceptionnel dans le système naturaliste du nominalisme. Or, précisément, de la théorie ockhamienne sur la foi, les nominalistes ultérieurs ne retiendront que cette exception au naturalisme du système, exception qu'ils rejetteront (R. Holcot) ou nuanceront (P. d'Ailly). Robert Holcot, en effet, dominicain, oxonien, nominaliste et ockhamiste, se sépare du Venerabilis inceptor, son contemporain, sur cette exception volontariste de l'acte de foi (chapitre 3). Sa critique radicalise l'opposition entre naturalisme et volontarisme. Clairement, la volonté n'a pas de pouvoir causal sur l'assentiment au vrai. Pour lui, toute croyance dépend de raisons motrices sur lesquelles l'esprit n'a pas directement prise et qui inscrivent l'acte d'assentiment dans un processus naturel. D'un mot, l'esprit est agi. La volonté n'intervient que pour orienter l'intellect en vue de chercher des motifs de croire. De cause première, la volonté a été détrônée au statut de cause concourante (concausa). Troisième temps, celui des synthèses (chapitre 4). Pierre d'Ailly introduit la notion-clé de pia affectio pour articuler le volontarisme d'Ockham au naturalisme d'Holcot. C'est la volonté pieusement affectée (voluntas pie affectata ou pia affectio voluntatis) qui peut commander l'intellect. Ainsi, il rejette le volontarisme strict d'Ockham et le fait que la volonté soit cause exclusive de l'assentiment de foi. Pour lui, l'assentiment de foi est dû à deux causes concourantes: un argument probable (le témoignage ou l'autorité) et l'affection pieuse (ou commandement de la volonté). Ce proche de Jean Gerson redirait à sa manière la dimension affective et non pas seulement intellectuelle de la foi. Le premier mouvement de l'intellect serait achevé dans la volonté en tant qu'elle est affectée pieusement: il s'agit d'un volontarisme modéré. Quant à Jean Mair, nominaliste parisien du début du XVI ${ }^{\mathrm{e}}$ siècle, il recentre, plus encore que ne l'a fait Pierre d'Ailly, la solution en élaborant une authentique voie moyenne: la foi ne peut ni relever d'un assentiment contraint par des arguments naturellement évidents, ni avoir pour cause évidente la volonté. Sa synthèse réside en 
deux mots : la volonté libre est nécessaire pour que l'acte de foi soit en notre pouvoir mais insuffisante pour lever par elle-même le doute sur une proposition. Le premier acte de foi est donc à la fois libre et motivé. Ce volontarisme mitigé, appuyé sur l'affection pieuse de Pierre d'Ailly, s'inscrit dans la filiation ockhamienne pour qui l'acte de volonté libre était au fondement de la foi, en lui ôtant son exclusivité : la volonté n'est que cause concourante qui accompagne le témoignage ou l'argument topique. La synthèse hérite donc des limites posées par Holcot au volontarisme ockhamien.

De cette démonstration, Christophe Grellard tire des interprétations historiques, et c'est ici que s'ouvre pour nous la discussion. Comment à partir de cette reconstitution des débats, l'auteur peut-il inférer que l'on est passé d'une conception médiévale à une conception moderne de la foi ? En réalité, son intuition part du fondamental article sur la « formalité des pratiques » de Certeau, dont, visiblement, la conclusion l'a séduit. Or, il entend étayer cette conclusion par les changements théoriques et doctrinaux de la fin du Moyen Âge, sous-évalués et sous-utilisés selon lui dans le projet certalien. L'ambition était claire: Christophe Grellard entendait éclairer et renforcer le fameux passage d'un système de croyance médiéval à un vécu religieux moderne, le passage d'une foi comme phénomène objectif à la foi comme phénomène subjectif, écrit-il. Autrement dit, il reprend le dossier sous l'angle d'une histoire des doctrines : " La thèse de Certeau me semble fondamentalement correcte, [...] et l'un des moyens d'évaluer les évolutions qu'il décrit, c'est de prendre en compte avant tout les doctrines. [...] Le débat nominaliste auquel on va s'intéresser est ici une partie de cette histoire doctrinale qui examine les changements théoriques rendant possibles les évolutions identifiées par Certeau " (p. 15). Ceci posé, le souci chez Christophe Grellard, d'une méthodologie historique, dûment réfléchie, appliquée aux textes philosophiques et aux constructions doctrinales, est louable. Mieux, il est prescriptif voire directif. Pour autant, l'on ne voit pas, dans sa mise en œuvre, que cette manière d'écrire l'histoire des doctrines soit adossée à une authentique anthropologie du croire, laquelle seule aurait pu inférer la perception d'une modernité à l'échelle d'une société. L'auteur soupçonne la difficulté de son projet (p.15-16) mais ne s'y arrête pas. Ce saut, pourtant véritable décrochement interprétatif, entre les discours normatifs des doctrines et le monde de la pratique ne pouvait s'opérer dans le seul cadre d'une enquête doxographique. Car, disons-le, les doctrines ne peuvent à elles seules induire les mutations sociales et l'historien ne peut, par la seule étude des doctrines, rendre compte des changements de paradigme d'une société. Là est le leurre. Sinon à risquer le schématisme, non, le nominalisme n'est pas le symptôme d'une individualisation de la vie religieuse (p. 16). Pour prétendre, en effet, discerner le passage d'une foi médiévale à une foi moderne, il eût fallu plus largement capter la circulation des doctrines dans les milieux de la pratique, dans les sources pastorales, dans les mises en œuvres vernaculaires, dans les réceptions des différents lectorats, pour ne donner que quelques exemples. Il eût fallu cerner les indices de l'acculturation des doctrines aux pratiques, les formes de cet évidement, dont parle si bien Michel de Certeau, cet évidement de sens des pratiques qui deviennent formalités, en contraste avec le vécu profond qui s'en est imperceptiblement détaché. Encore une fois, Certeau aura fasciné mais n'en est pas disciple qui veut. Si, en effet, la rupture que pose Certeau entre Moyen Âge et modernité se situe aux XVII ${ }^{\mathrm{e}}$ et XVIII ${ }^{\mathrm{e}}$ siècles, c'est parce qu'il observe ce moment où le rapport se défait entre les conduites et les convictions, entre les comportements religieux et la foi intime, entre le conformisme et l'intériorité. Au sens strict, il y a loin de ce divorce à la "sécularisation" (p.14), terme anachronique et téléologique, 
réducteur sans conteste. Il eût fallu encore, pour mieux cerner la «modernité » dont parle Certeau, s'interroger sur l'acception, au cœur du titre et du livre, de la foi comme certitude volontaire. Or, tous les écrits du jésuite ne disent rien d'autre que la faiblesse du croire (1987), cette suspension de la foi au-dessus du vide. Dans l'acception centralienne, la croyance religieuse s'avère tout le contraire d'une certitude. Et si le vrai passage à la modernité résidait dans cette authentique mutation? La foi non plus entendue comme une obédience mais comme un cheminement, non plus un refuge mais une aventure, non pas une certitude mais un risque. Une «itinérance", disait Certeau. Précisément, à l'époque moderne, la foi se définit par cette confiance qui n'a pas la garantie de ce qui la fonde, et qui ne se confond plus avec la solidité des institutions. De l'avènement de la modernité, les débats nominalistes ne pouvaient rendre compte, du moins, à eux seuls.

\section{AUTEURS}

\section{BÉNÉDICTE SÈRE}

Université Paris Ouest - Nanterre La Défense. 